2

\title{
Constitutive G protein coupling profiles of understudied orphan GPCRs
}

Sumin Lu ${ }^{1}$, Wonjo Jang ${ }^{1}$, Asuka Inoue ${ }^{2}$ and Nevin A. Lambert ${ }^{1}$

${ }^{1}$ Department of Pharmacology and Toxicology, Medical College of Georgia, Augusta University, Augusta, GA 30912, USA

${ }^{2}$ Graduate School of Pharmaceutical Sciences, Tohoku University, Sendai, Japan

Correspondence:

Nevin Lambert

1460 Laney Walker Boulevard

Augusta, Georgia 30912

USA

706-721-6336

E-mail: nelambert@augusta.edu.

\section{ORCID:}

Wonjo Jang: https://orcid.org/0000-0002-1928-8978

Nevin Lambert: https://orcid.org/0000-0001-7550-0921

Author contributions: S.L. and W.J. carried out all experimental procedures. A.I. generated and validated the G protein-deficient cell line. S.L., W.J. and N.L. analyzed and interpreted data. N.L. prepared figures and drafted the manuscript. All authors reviewed, revised, and approved the manuscript for publication. 


\section{Abstract}

29 A large number of GPCRs are potentially valuable drug targets but remain understudied. Many

30 of these lack well-validated activating ligands and are considered "orphan" receptors, and G

31 protein coupling profiles have not been defined for many orphan GPCRs. Here we asked if

32 constitutive receptor activity can be used to determine $\mathrm{G}$ protein coupling profiles of orphan

33 GPCRs. We monitored nucleotide-sensitive interactions between 48 understudied orphan

34 GPCRs and five $\mathrm{G}$ proteins (240 combinations) using bioluminescence resonance energy

35 transfer (BRET). No receptor ligands were used, but GDP was used as a common $\mathrm{G}$ protein

36 ligand to disrupt receptor-G protein complexes. Constitutive BRET between the same receptors

37 and $\beta$-arrestins was also measured. We found sufficient GDP-sensitive BRET to generate $G$

38 protein coupling profiles for 22 of the 48 receptors we studied. Altogether we identified 48

39 coupled receptor-G protein pairs, many of which have not been described previously. We

40 conclude that receptor-G protein complexes that form spontaneously in the absence of guanine

41 nucleotides can be used to profile $G$ protein coupling of constitutively active GPCRs. This

42 approach may prove useful for studying $\mathrm{G}$ protein coupling of other GPCRs for which activating 43 ligands are not available. 


\section{Introduction}

46 G protein-coupled receptors (GPCRs) are the targets of a large fraction of clinically-useful

47 drugs, and efforts to develop new drugs targeting GPCRs are ongoing [1]. Defining

48 characteristics of GPCRs are the natural ligands that bind and activate each receptor, and the

49 intracellular transducers (G proteins and arrestins) that propagate signals to downstream

50 effectors [2]. Individual GPCRs can couple to several different G proteins from more than one $G$

51 protein family. Because each of the four $G$ protein families $\left(G_{s / \text { olf }}, G_{i / 0}, G_{q / 11}\right.$, and $\left.G_{12 / 13}\right)$ activates

52 different downstream effectors, GPCR-G protein coupling profiles have traditionally been

53 determined using second messenger assays, most commonly those that measure intracellular

54 cyclic AMP (cAMP) and calcium. Although these assays are robust and quite sensitive, crosstalk between pathways can complicate interpretation, and comparable second messenger assays are not available for all four families. G protein coupling can also be determined by more direct methods, such as $\left[{ }^{35} \mathrm{~S}\right] \mathrm{GTP} \gamma \mathrm{S}$ binding in vitro, but these methods are more difficult to implement, particularly at scale across multiple $G$ protein subtypes [3]. More recently, genetic, spectroscopic and luminometric assays have been developed that allow more direct assessment of $G$ protein coupling profiles in living cells [4-8]. These assays can detect coupling to all four $\mathrm{G}$ protein families, avoid ambiguity due to signal crosstalk, and are efficient enough to allow profiling of a large number of GPCRs in parallel.

Several recent studies have used these methods to profile $G$ protein coupling of GPCRs in response to activating ligands [9-12]. However, for a large number of so-called orphan GPCRs the natural ligand is either not known or not well-validated, and surrogate activating ligands are not available [13]. Therefore, studies profiling $G$ protein coupling have generally not included orphan GPCRs. In a recent study we found that many GPCRs would spontaneously form complexes with cognate $\mathrm{G}$ proteins in the absence of guanine nucleotides, and these complexes were disrupted by the addition of GDP [14]. This is consistent with the known ability of constitutively-active GPCRs to activate $\mathrm{G}$ proteins in the absence of an agonist [15]. It occurred to us that the nucleotide-sensitivity of spontaneous GPCR-G protein complexes could be used to define coupling profiles of orphan GPCRs without using activating ligands. Here we test this idea using 48 orphan GPCRs, most of which have not been extensively studied or characterized. We find that approximately half of the receptors we studied possess sufficient constitutive activity to define a G protein coupling profile. These results may facilitate efforts aimed at understanding the physiological roles these receptors, and at discovering and validating new drugs acting at GPCRs. 


\section{Materials and Methods}

\section{Materials}

83 Trypsin, culture media, PBS, DPBS, penicillin/streptomycin and L-glutamine were from GIBCO

84 (ThermoFisher Scientific, Waltham, MA, USA). PEI MAX was purchased from Polysciences Inc.

85 (Warrington, PA, USA). Digitonin, apyrase and GDP were purchased from MilliporeSigma (St.

86 Louis, MO, USA). Coelenterazine h was purchased from Nanolight Technologies (Pinetop, AZ, 87 USA).

\section{Plasmid DNA constructs}

GPCR coding sequences were provided by Bryan Roth (University of North Carolina, Chapel Hill, NC; PRESTO-Tango Kit - \#1000000068, Addgene, Watertown), MA, USA) [16], except for GPR139, which was a gift from Kirill Martemyanov [17]. For each receptor the coding sequence was amplified with a common forward primer (corresponding to a cleavable signal sequence) and custom reverse primer (corresponding to the receptor $\mathrm{C}$ terminus) and ligated into a pRluc8-N1 cloning vector. All plasmid constructs were verified by Sanger sequencing. Plasmids encoding Venus-Kras, Venus-PTP1b, Venus-1-155-G $\gamma_{1}$, and Venus-155-239-G $\beta_{1}$ have been described previously $[4,18]$. $\mathrm{G} \alpha$ subunit plasmids were purchased from cdna.org (Bloomsburg University, Bloomsburg, PA). Plasmids encoding Venus- $\beta$-arrestin-1 and -2 were a gift from Vsevolod Gurevich (Vanderbilt University, Nashville, TN, USA), and plasmids encoding the S1 subunit of pertussis toxin (PTX-S1) was kindly provided by Stephen R. Ikeda (NIAAA, Rockville, MD, USA).

\section{Cell culture and transfection}

HEK 293 cells (CLS Cat\# 300192/p777_HEK293, RRID:CVCL_0045; ATCC, Manassas, VA, USA) were propagated in plastic flasks and on 6-well plates according to the supplier's protocol.

112 Ga subunit, Venus-1-155-G $\gamma_{2}$, Venus-155-239-G $\beta_{1}$, and pcDNA3.1(+) or PTX-S1 in a

$113(1: 10: 5: 5: 5)$ ratio for a total of $2.6 \mu \mathrm{g}$ of plasmid DNA in each well of a 6 -well plate. For arrestin 
114 experiments HEK 293 cells were transfected with a GPCR-Rluc8, Venus- $\beta$-arrestin-1 or -2,

115 GRK2 and GRK6 in a 1:10:5:5 ratio for a total of $2.1 \mu \mathrm{g}$ of plasmid DNA. For trafficking

116 experiments HEK 293 cells were transfected with a GPCR-Rluc8 and either Venus-Kras or

117 Venus-PTP1b in a 1:10 ratio for a total of $1.1 \mu \mathrm{g}$ of plasmid DNA.

\section{BRET assays}

120 For $\mathrm{G}$ protein coupling experiments cells were washed twice with permeabilization buffer (KPS)

121 containing $140 \mathrm{mM} \mathrm{KCl}, 10 \mathrm{mM} \mathrm{NaCl}, 1 \mathrm{mM} \mathrm{MgCl}$, $0.1 \mathrm{mM} \mathrm{KEGTA}, 20 \mathrm{mM}$ NaHEPES (pH

122 7.2), harvested by trituration, permeabilized in KPS buffer containing $10 \mu \mathrm{g} \mathrm{ml}^{-1}$ high purity

123 digitonin, and transferred to opaque black 96-well plates. Measurements were made from

124 permeabilized cells supplemented either with $100 \mu \mathrm{M} \mathrm{GDP}$ or $2 \mathrm{U} \mathrm{ml}^{-1}$ apyrase. For arrestin and

125 trafficking experiments cells were washed twice in PBS and harvested by trituration in DPBS.

126 For all experiments $5 \mu \mathrm{M}$ coelenterazine $\mathrm{h}$ was used as a substrate. For the experiments shown

127 in Fig 1, permeabilized cells were supplemented with apyrase, and GDP (100 $\mu \mathrm{M})$ was injected

128 during continuous recording using a Polarstar Optima plate reader (BMG Labtech, Offenburg,

129 Germany). All other measurements were made using a Mithras LB940 photon-counting plate

130 reader (Berthold Technologies $\mathrm{GmbH}$, Bad Wildbad, Germany). Raw BRET signals were

131 calculated as the emission intensity at $520-545 \mathrm{~nm}$ divided by the emission intensity at 475-495

$132 \mathrm{~nm}$. Net BRET signals were calculated as the raw BRET signal minus the raw BRET signal

133 measured from cells expressing only the Rluc8 donor.

\section{Statistical analysis}

136 The data shown in Fig 1 represent the mean \pm SD of 16 technical replicates from one exemplary experiment. Because background basal BRET differed for each $\mathrm{G}$ protein, in this experiment raw BRET values for each trace are normalized the average of the first ten data points of all of the traces for a particular $G$ protein. The data shown in Fig 2 represent the average of three independent experiments, each performed in duplicate. G protein heat maps (Fig 3) represent

141 the difference in the raw BRET ratios measured from cells incubated in presence and absence

142 of GDP ( $\triangle B_{\text {RET }}$ GDP). Arrestin heat maps represent the basal net BRET. No hypothesis testing 143 was performed and no claims of statistical significance are made. The threshold for assigning G 144 protein coupling was determined by assuming that the majority of receptor-G protein pairs would 145 be uncoupled, and that the $\triangle B R_{G D P}$ values for these pairs would be randomly distributed 146 around zero. Coupled pairs were detected as outliers from this distribution using the ROUT 
147 method [20] implemented in GraphPad Prism 8 (GraphPad Software, La Jolla, CA) with Q (the

148 maximum false discovery rate) set to $1 \%$, meaning fewer than $1 \%$ of the detected (coupled)

149 pairs are expected to be false-positives. The same procedure was used to detect receptor-

150 arrestin pairs. 


\section{Results}

\section{Receptor-G protein interactions}

154 We have previously monitored direct interactions between GPCRs and G proteins using 155 bioluminescence resonance energy transfer (BRET) between receptors fused to Renilla

156 luciferase (Rluc8) and G protein heterotrimers tagged with the fluorescent protein Venus. Using 157 permeabilized cells we found that many GPCRs spontaneously interacted with G proteins in a 158 nucleotide-sensitive fashion [14]. Importantly, these constitutive GDP-sensitive interactions 159 corresponded well to known G protein coupling, suggesting that it should be possible to study G 160 protein coupling of orphan GPCRs without using activating ligands. To test this idea we fused 161 Rluc8 to the $C$ terminus of 48 class $A$ orphan receptors, 43 of which are on the most recent list 162 of understudied GPCR targets compiled by the Illuminating the Druggable Genome (IDG) project $[21,22]$. Receptors were coexpressed together with a $\mathrm{G} \alpha$ subunit and Venus-G $\beta \gamma$ in genome-edited HEK 293 cells lacking endogenous $G_{s / \text { off }} G_{q / 11}$ and $G_{12 / 13}$ proteins [19]. We chose one $G \alpha$ subunit to represent each of the four $G$ protein families $\left(G \alpha_{i 1}, G \alpha_{s}\right.$-long, $G \alpha_{q}$,

$\left.166 \mathrm{G} \alpha_{13}\right)$ as well as $\mathrm{G} \alpha_{15}$, due to its unique coupling properties [23]. Except when $\mathrm{G} \alpha_{i 1}$ was used, 167 we also transfected the $\mathrm{S} 1$ subunit of pertussis toxin to prevent coupling of endogenous $\mathrm{G}_{\mathrm{i} / \mathrm{o}}$ 168 proteins to GPCRs.

Complexes between constitutively-active receptors and cognate $G$ proteins formed spontaneously in permeabilized cells when apyrase was used to hydrolyze residual guanine nucleotides, thus maintaining the nucleotide-empty state of the G protein. Addition of GDP (100 $\mu \mathrm{M})$ led to rapid complex dissociation, and a decrease in BRET between receptors and $G$

174 proteins (Fig 1A). For example, GPR82 formed GDP-sensitive complexes with $\mathrm{G}_{i 1}$ heterotrimers, 175 but not with $\mathrm{G}_{\mathrm{s}}, \mathrm{G}_{\mathrm{q}}$, or $\mathrm{G}_{13}$ heterotrimers (Fig 1B). In contrast, GPR174 formed GDP-sensitive complexes primarily with $\mathrm{G}_{\mathrm{s}}$ and $\mathrm{G}_{13}$ heterotrimers (Fig 1B). Almost nothing is known about GPR82 (Jensen PubMed Score 1.16), which is listed as a "probable" GPCR, and we were

178 unable to find any reports of GPR82 coupling to G proteins in the literature. In contrast, GPR174

179 (Jensen PubMed Score 10.06) has been described as a receptor for lysophosphatidyl-L-serine

180 (lysoPS) [7] and the chemokine CCL21 [24], and is known to couple to $\mathrm{G}_{\text {s/olf }}$ and $\mathrm{G}_{12 / 13}$

181 heterotrimers [9]. These results demonstrate the utility of this approach for profiling $\mathrm{G}$ protein

182 coupling of orphan GPCRs. 
Changes in BRET after addition of GDP ( $\left.\triangle B R E T_{G D P}\right)$ for the 240 pairings in our sample clustered around zero, as expected if the majority of receptor-G protein pairs do not constitutively couple (Fig 2). However, a population of more negative values of $\triangle B R E T_{\text {GDP }}$ was apparent that presumably corresponds to coupled receptor-G protein pairs. We set a conservative threshold for coupling by identifying outliers from a random distribution of $\triangle$ BRET $_{G D P}$ values, using a false discovery rate (FDR) of $1 \%$ (see Materials and Methods).

Using this threshold $\mathrm{G}$ protein coupling was detected for 22 of the 48 receptors and 48 of the 240 pairings in our sample (Fig 3; S1 File). We detected constitutive coupling of 18 receptors to $G_{i 1}, 8$ receptors to $G_{s}, 6$ receptors to $G_{q}, 7$ receptors to $G_{13}$, and 9 receptors to $G_{15}$. Of the 22 profiled receptors 11 are annotated for G protein coupling in the IUPHAR Guide to Pharmacology (GtoPdb), and within this set there was excellent agreement between our results and annotated coupling [13] (S1 File). The sole exception was GPR75, which coupled to $\mathrm{G}_{\mathrm{i} 1}$ in our dataset but is annotated as coupling to $G_{q}$. This receptor has been shown to stimulate inositol phosphate turnover and calcium release in other studies [25]. In several cases, our results agreed with annotated receptor-G protein pairs, but also indicated coupling to additional

$200 \mathrm{G}$ protein families. For example, our results confirmed coupling of $\mathrm{GPR} 26$ to $\mathrm{G}_{\mathrm{s}}$ [26], but 201 indicated additional coupling to $G_{11}, G_{15}$ and $G_{q}$. We also found several instances where no $G$ protein coupling was annotated in GtoPdb, but where published reports indicated signaling through a particular $G$ protein pathway. In such cases our results were also generally in good agreement with previous reports, but again indicated coupling to additional G proteins that was previously unreported. For example, GPR62 has been shown to constitutively activate adenylyl cyclase (AC), albeit weakly, suggesting coupling to $G_{s}$ [27]. Our results confirm that this receptor couples to $G_{s}$, but also show similar coupling to $G_{i 1}$ and $G_{15}$. Dual coupling to $G_{i}$ and $G_{s}$ proteins may help to explain relatively weak constitutive activation of $A C$ by this receptor. These results illustrate the value of an unbiased profiling approach that includes $G$ proteins from all four $G \alpha$

210 subunit families.

212 For 26 of the receptors we studied $\triangle B R E T_{G D P}$ did not meet threshold for any of the $G$ proteins

213 tested. The most likely explanation for this outcome is that these receptors simply lacked

214 sufficient constitutive activity to couple efficiently to $G$ proteins in the absence of a ligand.

215 However, one alternative explanation is the failure of these receptors to traffic efficiently to the 216 plasma membrane, where the majority of $G$ protein heterotrimers are located. To test this idea 217 we measured bystander BRET between each receptor and markers of the plasma membrane 
218 (PM) and endoplasmic reticulum (ER) [18]. Most receptors showed substantial BRET to the PM

219 marker, and less BRET to the ER marker, indicating efficient trafficking to the cell surface.

220 However, 8 receptors (GPR31, GPR37L1, GPR142, GPR146, GPR148, GPR152, GPR160 and

221 MRGPRG) showed BRET to the ER marker that exceeded BRET to the PM marker, indicating

222 inefficient trafficking to the PM (S1 File). All 8 of these receptors were among the 26 that failed

223 to show constitutive $G$ protein coupling, suggesting that retention of these receptors in the

224 biosynthetic pathway may have contributed to our inability to detect G protein coupling.

\section{Receptor-arrestin interactions}

227 It is also possible that some of the receptors that we studied do not couple to $\mathrm{G}$ proteins at all, 228 as is the case for some "decoy" receptors (e.g. the C5a2 complement receptor) [28]. Because 229 some decoy receptors bind to $\beta$-arrestins we asked if any of the orphan receptors in our sample 230 interacted constitutively with these transducers by measuring basal BRET between receptors 231 and Venus- $\beta$-arrestin- 1 and Venus- $\beta$-arrestin-2 in intact cells. Basal BRET between

232 unstimulated GPCRs and arrestins is typically low unless there is a specific interaction [29], or 233 unless arrestins are recruited in some other way to membrane compartments where receptors 234 are located. Accordingly, basal BRET between orphan receptors and Venus- $\beta$-arrestins was low 235 for most of the receptors in our sample (Fig 3; S1 File). However, GPR182 and GPR4 were both 236 outliers for both $\beta$-arrestin-1 and $\beta$-arrestin-2. GPR182 failed to couple detectably to $\mathrm{G}$ proteins, 237 suggesting that this receptor may be biased towards interacting with arrestins rather than $\mathrm{G}$ 238 proteins. 


\section{Discussion}

241 In the present study we measured guanine nucleotide-sensitive coupling of $\mathrm{G}$ proteins to a

242 sample of understudied orphan GPCRs. We used an unbiased approach that directly indicates

243 receptor association with unmodified $\mathrm{G} \alpha$ subunits and does not require an activating ligand. We 244 were able to detect $\mathrm{G}$ protein coupling to 22 of the 48 receptors we studied. We confirmed many receptor-G protein pairings determined previously by other methods, and demonstrated several new pairings. With respect to the overall prevalence of coupling to different $G$ protein subtypes, our results with constitutive activity of orphan receptors agree well with previous studies of agonist-induced coupling of non-orphan GPCRs [9-12]. $\mathrm{G}_{i 1}$ was the most frequent coupler (18 receptors), whereas the $G_{q / 11}$ family (including $G_{q}$ and $G_{15}$ ) was the second-most frequent (15 receptors). Of the 9 receptors that coupled to only one $G$ protein, 7 coupled solely to $G_{i 1}$. We also found that coupling to $\mathrm{G}_{13}$ (7 receptors) was more common in our dataset than might be predicted based on GtoPdb annotations of all GPCRs, as shown previously by others $[9,10]$. It is possible that coupling to $\mathrm{G}_{12 / 13}$ is underrepresented in GtoPdb because simple second messenger assays are not available for this family. Coupling to $G_{13}$ was always observed in conjunction with coupling to another $\mathrm{G}$ protein [30]. Among the $\mathrm{G}_{13}$-coupled receptors in our sample were all 4 members of a closely-related family of acid-sensing receptors (GPR4, GPR65, GPR68 and GPR132) [31], all of which coupled to $G_{13}$ at least as well as any other $G$

258 protein.

The assay that we used here has a particular advantage for studies of constitutive receptor activity, in that GDP can essentially be used as a common ligand to disrupt coupled GPCR-G protein complexes. This comes with a significant drawback, in that constitutive activity is required, and a subjective threshold was needed to assign receptor-G protein coupling. It is

264 likely that many of the receptors that we were unable to profile will couple well to $G$ proteins 265 when bound to an activating ligand. Although these caveats mean that our study undoubtedly

266 missed several receptor-G protein pairings, it also suggests that our results can help predict 267 which of these orphan receptors have high and low constitutive activity. For example, GPR18 is 268 a relatively well-studied receptor (Jensen PubMed Score 42.64) that binds to endogenous 269 cannabinoid compounds [32] and is annotated in GtoPdb as coupling to $G_{i / o}$ and $G_{q / 11}$. This 270 receptor showed subthreshold $\triangle B R E T_{G D P}$ (which was greatest for $G_{i 1}$ ) in our study, suggesting 271 that GPR18 has low constitutive activity compared to other receptors in our sample. Another 272 limitation of our study is that we did not address selectivity among G proteins within a family, 273 although this could be easily rectified with additional studies. We also identified several orphan 
274 receptors that are at least partly retained in the endoplasmic reticulum of HEK 293 cells. These

275 receptors may require cell type-specific trafficking factors to reach the plasma membrane. For

276 example, GPR37L1 is expressed almost exclusively in glial cells and is thought to couple to $\mathrm{G}_{\mathrm{i} / \mathrm{o}}$

277 proteins [33], but trafficked poorly to the cell surface in HEK 293 cells.

279 We found one receptor, GPR182, that did not couple to G proteins in our assay, but did

280 constitutively interact with $\beta$-arrestins. This result is consistent with a previous study that

281 showed very high constitutive binding of a GPR182-V2R vasopressin receptor fusion protein to

$282 \beta$-arrestin [16]. Gene-transcription studies suggest that this receptor may also signal through

283 several canonical G protein pathways [34], but specific $G$ protein coupling has not been

284 reported. Given the demonstrated importance of GPR182 for cellular proliferation and

285 hematopoiesis [35,36], our results suggest that further studies of GPR182 signaling

286 mechanisms are warranted.

In summary, we were able to profile constitutive $G$ protein coupling for a significant fraction of understudied class A orphan GPCRs. The success of this strategy suggests that it may be useful for profiling G protein coupling of other GPCRs (e.g. adhesion receptors and class C orphans) for which well-validated activating ligands are not available.

Acknowledgements: We thank Steve Ikeda, Seva Gurevich, Kirill Martemyanov and Bryan

295 Roth for providing plasmid DNA used in this study. This work was supported by a grant from the 296 National Institutes of Health (GM130142 to N.A.L.). A.I. was funded by the PRIME

297 JP19gm5910013, the LEAP JP19gm0010004 and the BIND JP19am0101095 from the Japan

298 Agency for Medical Research and Development (AMED). 
300

301

302

303

304

305

306

307

308

309

310

311

312

313

314

315

316

317

318

319

320

321

322

323

324

325

326

327

328

329

330

331

332

333

334

335

336

337

338

339

340

341

342

343

344

345

346

347

348

349

350

\section{References:}

1. Hauser AS, Attwood MM, Rask-Andersen M, Schiöth HB, Gloriam DE. Trends in GPCR drug discovery: New agents, targets and indications. Nat Rev Drug Discov. 2017;16: 829-842. doi:10.1038/nrd.2017.178

2. Pierce KL, Premont RT, Lefkowitz RJ. Seven-transmembrane receptors. Nat Rev Mol Cell Biol. 2002;3: 639-650.

3. Milligan G. Principles: Extending the utility of [35S]GTPYS binding assays. Trends in Pharmacological Sciences. Trends Pharmacol Sci; 2003. pp. 87-90. doi:10.1016/S01656147(02)00027-5

4. Hollins B, Kuravi S, Digby GJ, Lambert NA. The c-terminus of GRK3 indicates rapid dissociation of $G$ protein heterotrimers. Cell Signal. 2009;21: 1015-1021. Available: http://www.ncbi.nlm.nih.gov/entrez/query.fcgi?cmd=Retrieve\&db=PubMed\&dopt=Citation \&list_uids $=19258039$

5. Galés C, Van Durm JJJ, Schaak S, Pontier S, Percherancier Y, Audet M, et al. Probing the activation-promoted structural rearrangements in preassembled receptor- $G$ protein complexes. Nat Struct Mol Biol. 2006;13: 778-786. doi:10.1038/nsmb1134

6. Gales C, Rebois R V, Hogue M, Trieu P, Breit A, Hebert TE, et al. Real-time monitoring of receptor and G-protein interactions in living cells. Nat Methods. 2005;2: 177-184.

Available:

http://www.ncbi.nlm.nih.gov/entrez/query.fcgi?cmd=Retrieve\&db=PubMed\&dopt=Citation \&list_uids $=15782186$

7. Inoue A, Ishiguro J, Kitamura H, Arima N, Okutani M, Shuto A, et al. TGFa shedding assay: An accurate and versatile method for detecting GPCR activation. Nat Methods. 2012;9: 1021-1029. doi:10.1038/nmeth.2172

8. Wan Q, Okashah N, Inoue A, Nehmé R, Carpenter B, Tate CG, et al. Mini G protein probes for active $G$ protein-coupled receptors (GPCRs) in live cells. J Biol Chem. 2018;293: 7466-7473. doi:10.1074/jbc.RA118.001975

9. Inoue A, Raimondi F, Kadji FMN, Singh G, Kishi T, Uwamizu A, et al. Illuminating GProtein-Coupling Selectivity of GPCRs. Cell. 2019;177: 1933-1947.e25. doi:10.1016/j.cell.2019.04.044

10. Avet C, Mancini A, Breton B, Gouill C Le, Hauser AS, Normand C, et al. Selectivity Landscape of 100 Therapeutically Relevant GPCR Profiled by an Effector TranslocationBased BRET Platform. bioRxiv. 2020; 2020.04.20.052027. doi:10.1101/2020.04.20.052027

11. Masuho I, Ostrovskaya O, Kramer GM, Jones CD, Xie K, Martemyanov KA. Distinct profiles of functional discrimination among $\mathrm{G}$ proteins determine the actions of $\mathrm{G}$ proteincoupled receptors. Sci Signal. 2015;8: ra123-ra123. doi:10.1126/scisignal.aab4068

12. Kapolka NJ, Taghon GJ, Rowe JB, Morgan WM, Enten JF, Lambert NA, et al. Dcyfir: A high-throughput CRISPR platform for multiplexed $G$ protein-coupled receptor profiling and ligand discovery. Proc Natl Acad Sci U S A. 2020;117: 13117-13126. doi:10.1073/pnas.2000430117

13. Alexander SPH, Christopoulos A, Davenport AP, Kelly E, Mathie A, Peters JA, et al. THE CONCISE GUIDE TO PHARMACOLOGY 2019/20: G protein-coupled receptors. Br J Pharmacol. 2019;176: S21-S141. doi:10.1111/bph.14748

14. Okashah N, Wan Q, Ghosh S, Sandhu M, Inoue A, Vaidehi N, et al. Variable G protein determinants of GPCR coupling selectivity. Proc Natl Acad Sci U S A. 2019;116: 1205412059. doi:10.1073/pnas.1905993116

15. Seifert R, Wenzel-Seifert K. Constitutive activity of G-proteins-coupled receptors: Cause of disease and common property of wild-type receptors. Naunyn-Schmiedeberg's Archives of Pharmacology. Naunyn Schmiedebergs Arch Pharmacol; 2002. pp. 381-416. doi:10.1007/s00210-002-0588-0 
351

352

353

354

355

356

357

358

359

360

361

362

363

364

365

366

367

368

369

370

371

372

373

374

375

376

377

378

379

380

381

382

383

384

385

386

387

388

389

390

391

392

393

394

395

396

397

398

399

400

401

16. Kroeze WK, Sassano MF, Huang X-P, Lansu K, McCorvy JD, Giguère PM, et al. PRESTO-Tango as an open-source resource for interrogation of the druggable human GPCRome. Nat Struct Mol Biol. 2015;22: 362-9. doi:10.1038/nsmb.3014

17. Stoveken HM, Zucca S, Masuho I, Grill B, Martemyanov KA. The orphan receptor GPR139 signals via Gq/11 to oppose opioid effects. J Biol Chem. 2020;295. doi:10.1074/jbc.ac120.014770

18. Lan T-H, Liu Q, Li C, Wu G, Lambert NA. Sensitive and High Resolution Localization and Tracking of Membrane Proteins in Live Cells with BRET. Traffic. 2012;13. doi:10.1111/j.1600-0854.2012.01401.x

19. Grundmann M, Merten N, Malfacini D, Inoue A, Preis P, Simon K, et al. Lack of betaarrestin signaling in the absence of active $G$ proteins. Nat Commun. 2018;9: 341. doi:10.1038/s41467-017-02661-3

20. Motulsky HJ, Brown RE. Detecting outliers when fitting data with nonlinear regression - A new method based on robust nonlinear regression and the false discovery rate. BMC Bioinformatics. 2006;7: 123. doi:10.1186/1471-2105-7-123

21. Sheils TK, Mathias SL, Kelleher KJ, Siramshetty VB, Nguyen D-T, Bologa CG, et al. TCRD and Pharos 2021: mining the human proteome for disease biology. Nucleic Acids Res. 2021;49: D1334-D1346. doi:10.1093/nar/gkaa993

22. Rodgers G, Austin C, Anderson J, Pawlyk A, Colvis C, Margolis R, et al. Glimmers in illuminating the druggable genome. Nature Reviews Drug Discovery. Nature Publishing Group; 2018. pp. 301-302. doi:10.1038/nrd.2017.252

23. Offermanns S, Simon MI. Ga15 and ga16 couple a wide variety of receptors to phospholipase C. J Biol Chem. 1995;270: 15175-15180. doi:10.1074/jbc.270.25.15175

24. Zhao R, Chen X, Ma W, Zhang J, Guo J, Zhong X, et al. A GPR174-CCL21 module imparts sexual dimorphism to humoral immunity. Nature. 2020;577: 416-420. doi:10.1038/s41586-019-1873-0

25. Ignatov A, Robert J, Gregory-Evans C, Schaller HC. RANTES stimulates Ca 2+ mobilization and inositol trisphosphate (IP 3) formation in cells transfected with $\mathrm{G}$ proteincoupled receptor 75. Br J Pharmacol. 2006;149: 490-497. doi:10.1038/sj.bjp.0706909

26. Jones PG, Nawoschik SP, Sreekumar K, Uveges AJ, Tseng E, Zhang L, et al. Tissue distribution and functional analyses of the constitutively active orphan $G$ protein coupled receptors, GPR26 and GPR78. Biochim Biophys Acta. 2007;1770: 890-901. doi:10.1016/j.bbagen.2007.01.013

27. Oishi A, Karamitri A, Gerbier R, Lahuna O, Ahmad R, Jockers R. Orphan GPR61, GPR62 and GPR135 receptors and the melatonin MT2 receptor reciprocally modulate their signaling functions. Sci Rep. 2017;7. doi:10.1038/s41598-017-08996-7

28. Li XX, Lee JD, Kemper C, Woodruff TM. The Complement Receptor C5aR2: A Powerful Modulator of Innate and Adaptive Immunity. J Immunol. 2019;202: 3339-3348. doi:10.4049/jimmunol.1900371

29. Angers S, Salahpour A, Joly E, Hilairet S, Chelsky D, Dennis M, et al. Detection of beta 2-adrenergic receptor dimerization in living cells using bioluminescence resonance energy transfer (BRET). Proc Natl Acad Sci U S A. 2000;97: 3684-3689. Available: http://www.ncbi.nlm.nih.gov/entrez/query.fcgi?cmd=Retrieve\&db=PubMed\&dopt=Citation \&list_uids $=10725388$

30. Riobo NA, Manning DR. Receptors coupled to heterotrimeric $\mathrm{G}$ proteins of the $\mathrm{G} 12$ family. Trends Pharmacol Sci. 2005;26: 146-154. doi:10.1016/j.tips.2005.01.007

31. Tomura H, Mogi C, Sato K, Okajima F. Proton-sensing and lysolipid-sensitive G-proteincoupled receptors: A novel type of multi-functional receptors. Cellular Signalling. Cell Signal; 2005. pp. 1466-1476. doi:10.1016/j.cellsig.2005.06.002

32. Irving A, Abdulrazzaq G, Chan SLF, Penman J, Harvey J, Alexander SPH. Cannabinoid Receptor-Related Orphan G Protein-Coupled Receptors. Advances in Pharmacology. 
33. Meyer RC, Giddens MM, Schaefer SA, Hall RA. GPR37 and GPR37L1 are receptors for the neuroprotective and glioprotective factors prosaptide and prosaposin. Proc Natl Acad Sci U S A. 2013;110: 9529-9534. doi:10.1073/pnas.1219004110

34. Schmid CD, Schledzewski K, Mogler C, Waldburger N, Kalna V, Marx A, et al. GPR182 is a novel marker for sinusoidal endothelial differentiation with distinct GPCR signaling activity in vitro. Biochem Biophys Res Commun. 2018;497: 32-38. doi:10.1016/j.bbrc.2018.01.185

35. Kechele DO, Blue RE, Zwarycz B, Espenschied ST, Mah AT, Siegel MB, et al. Orphan Gpr182 suppresses ERK-mediated intestinal proliferation during regeneration and adenoma formation. J Clin Invest. 2017;127: 593-607. doi:10.1172/JCI87588

36. Kwon HB, Mackie DI, Bonnavion R, Mercier A Le, Helker CSM, Son T, et al. The Orphan G-Protein Coupled Receptor 182 Is a Negative Regulator of Definitive Hematopoiesis through Leukotriene B4 Signaling. ACS Pharmacol TransI Sci. 2020;3: 676-689. doi:10.1021/acsptsci.0c00020 
A

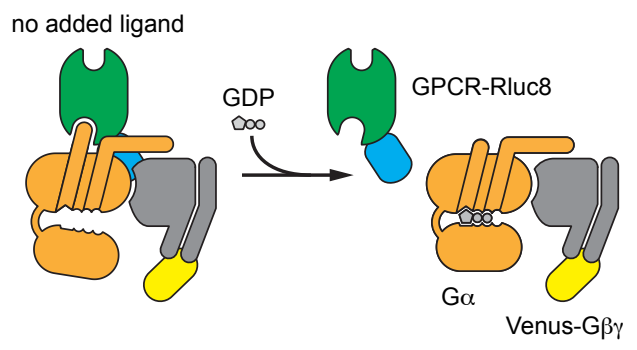

B

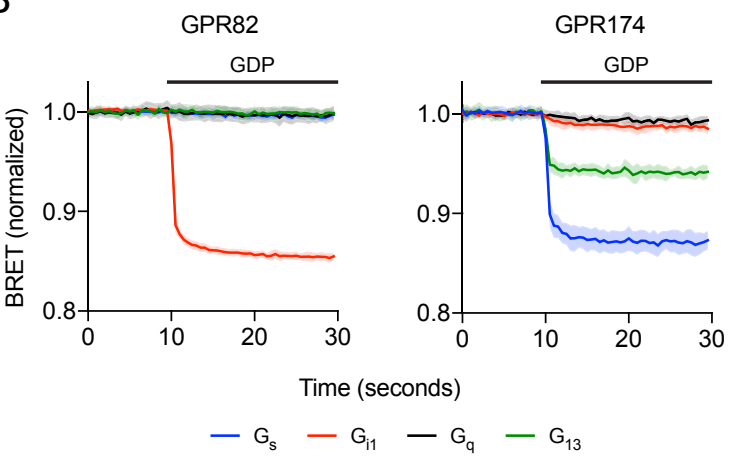

420 Fig 1. Addition of GDP disrupts GPCR-G protein complexes. (A) Cartoon representation of

421 the experimental design. Constitutively-active GPCRs fused to Renilla luciferase (Rluc8) form

422 spontaneous active-state complexes with nucleotide-free $\mathrm{G}$ protein heterotrimers fused (via the

$423 \mathrm{G} \beta \gamma$ subunit) to the fluorescent protein Venus in the absence of activating ligands. Addition of

424 GDP $(100 \mu \mathrm{M})$ disrupts these complexes, decreasing BRET between GPCR-Rluc8 and G $\alpha \beta \gamma$ -

425 Venus. (B) Representative experiments of this type with GPR82 and GPR174. Traces represent

426 the mean \pm SD of 16 technical replicates from a single experiment, and each trace is normalized

427 to the basal BRET observed for that particular G protein. GDP was injected where indicated by

428 the horizontal bar. 


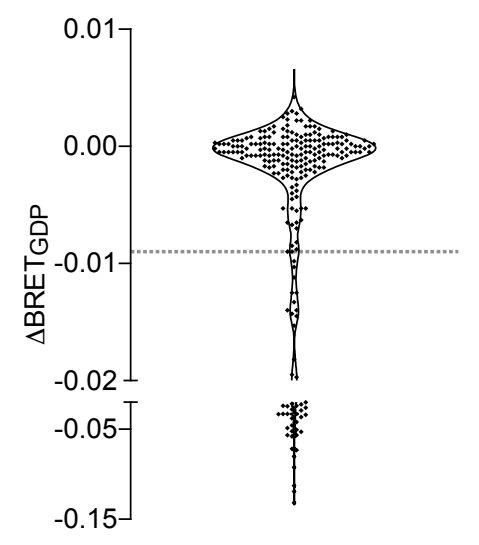

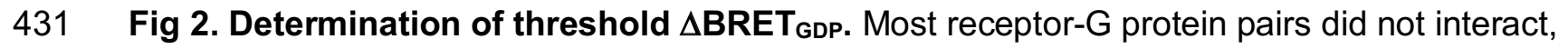

432 and values of $\triangle B R E T_{G D P}$ were distributed around zero. Values below the dashed horizontal line

$433\left(\triangle B R E T_{G D P}=-0.009\right)$ were identified as outliers from this background distribution (i.e. coupled

434 pairs) with a false discovery rate (FDR) of $1 \%$. Each point represents a single receptor-G protein

435 pair, and the mean of three independent experiments performed in duplicate. 


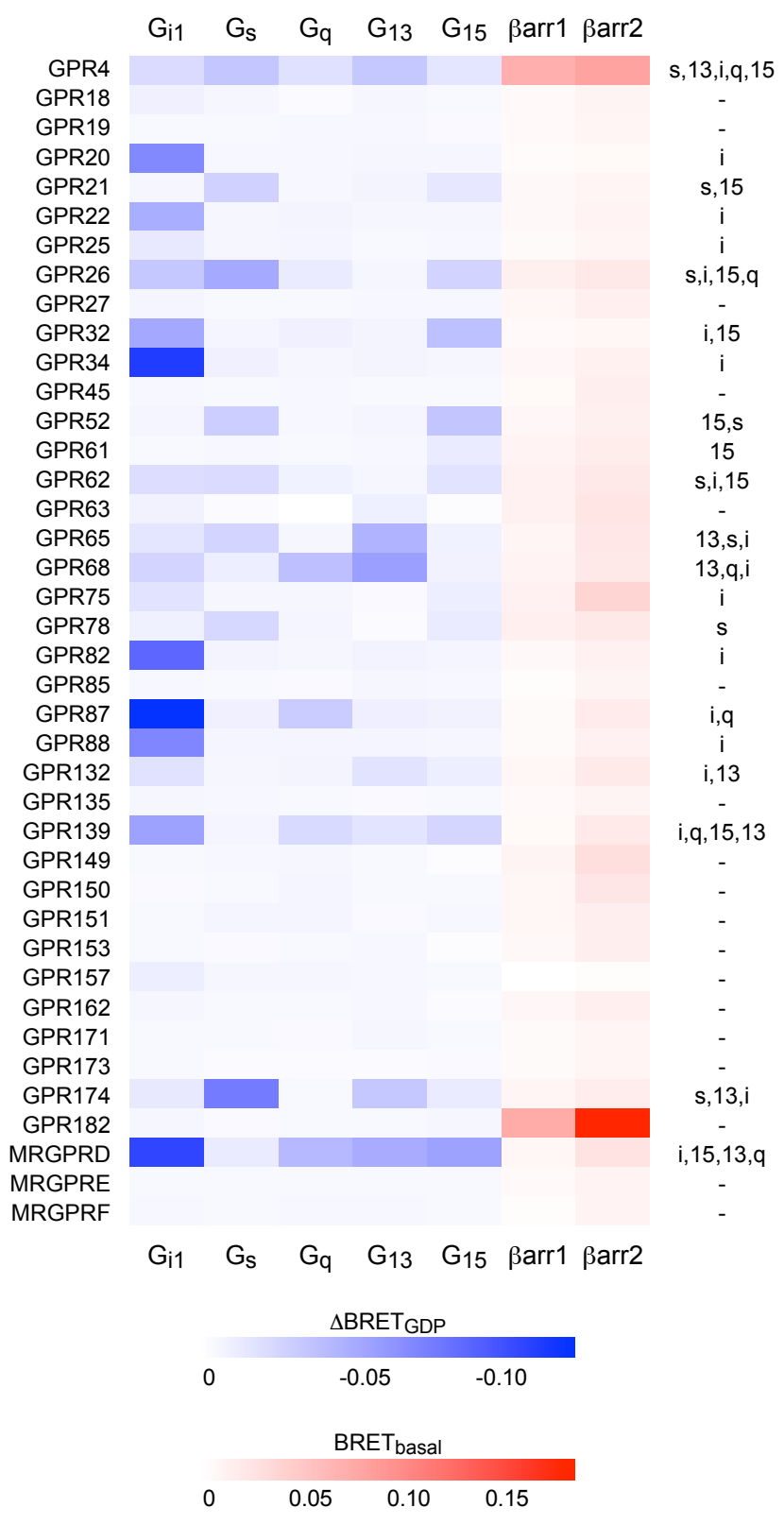

438 Fig 3. Constitutive $\mathbf{G}$ protein and $\beta$-arrestin coupling of understudied GPCRs. Heat maps

439 representing the mean $\triangle \mathrm{BRET}_{\mathrm{GDP}}$ for 200 receptor-G protein pairs (blue) and basal net BRET

440 for 80 receptor- $\beta$-arrestin pairs (red). The righthand column indicates the $\mathrm{G}$ proteins for which

$441 \triangle$ BRET $_{G D P}$ exceeded the determined threshold. Each cell represents the mean of three

442 independent experiments performed in duplicate. Eight receptors that trafficked poorly to the

443 plasma membrane are not shown here. 